\title{
Habilidades Cognitivas, Contexto Rural y Urbano: Comparación de Perfiles WAIS-IV en Jóvenes
}

\section{Cognitive Skills, Rural and Urban Context: Comparison of WAIS-IV Profiles in Youth}

\author{
Pablo Fuica \\ Juan Lira \\ Katia Alvarado \\ Camila Araneda \\ Gonzalo Lillo \\ Rocío Miranda
}

Departamento de Psicología, Facultad de Ciencias Sociales, Universidad de Concepción, Chile

Marcela Tenorio

Centro de Desarrollo de Tecnologías de Inclusión (CEDETi UC), Escuela de Psicología, Pontificia Universidad Católica de Chile

Claudia Paz Pérez-Salas

Departamento de Psicología, Facultad de Ciencias Sociales, Universidad de Concepción, Chile

Rec (16 de Abril de 2013) Acept (20 de Mayo de 2014)

\section{Resumen}

La reciente estandarización en Chile de la Escala Wechsler de Inteligencia para Adultos (WAIS), en su cuarta versión, obliga a investigar el impacto que condiciones culturales y ambientales pueden tener sobre el rendimiento intelectual de las personas. El presente estudio comparó el desempeño de jóvenes provenientes de sectores rurales y urbanos de la región del Biobío, segmentados según nivel educativo de los padres como variable de aproximación al nivel socioeconómico.

Los resultados muestran un efecto de interacción entre el nivel socioeconómico y el lugar de residencia. Comprensión verbal y memoria de trabajo son los constructos que reciben un mayor impacto del nivel socioeconómico. Se espera que futuros estudios contribuyan a la investigación de habilidades cognitivas en sectores rurales y socialmente deprivados de Chile.

Palabras clave: ruralidad, habilidades cognitivas, Escala Wechsler de Inteligencia para Adultos y deprivación social.

\begin{abstract}
The recent standardization of Wechsler Adult Intelligence Scale (WAIS) in its fourth version in Chile forces to test the impact that cultural and environmental conditions can have on people's intellectual performance. The present study compared the performance of young people from rural and urban areas of the Biobío region, segmented by parental education level as proxy variable to socioeconomic status.

Results showed an interaction effect between socioeconomic status and place of residence. Verbal Comprehension and Working Memory are the constructs that received a higher impact of the socioeconomic status. Future studies are expected to contribute to research on cognitive abilities in rural and socially deprived sectors in Chile.

Keywords: Rural environment, cognitive ability, Wechsler Adult Intelligence Scale, social deprivation.
\end{abstract}

Correspondencia:

Claudia Paz Pérez-Salas a cperezs@udec.cl o a la dirección Universidad de Concepción, Barrio Universitario, Facultad de Ciencias Sociales, Departamento de Psicología, segundo piso, Concepción, Chile. 


\section{Introducción}

\section{Habilidades cognitivas, ruralidad y acceso educativo}

David Wechsler (1979) definió la inteligencia como la capacidad de un individuo para actuar con una finalidad, pensar racionalmente y moverse con efectividad dentro de su ambiente. Sugirió que no es sólo una entidad global, sino que se trata de un conjunto de habilidades específicas, conocidas como habilidades cognitivas, que tienen una estrecha relación con el contexto.

Durante el siglo XX hubo una amplia difusión de las pruebas de coeficiente intelectual, hasta que las Escalas Wechsler se convirtieron en el estándar internacional aceptado (Strauss, Sherman y Spreen, 1999; Lichtenberger y Kaufman, 2009). Una característica de este instrumento, en sus diferentes versiones, ha sido la inclusión de una serie de subpruebas que exploran distintas habilidades y cuyo objetivo es obtener el perfil cognitivo del examinado.

La investigación ha mostrado que el contexto cultural genera diferencias significativas en los resultados que obtienen los sujetos en estas pruebas estandarizadas de evaluación cognitiva. Estas diferencias se han observado tanto en resultados generales como en perfiles específicos y han sido reportadas en función del nivel socio-económico, la zona de habitación (Flores-Mendoza y Nascimiento, 2007; Roazzi y Campello, 2002) y el nivel educativo (Flores-Mendoza y Nascimiento, 2007), entre otras variables.

Recientemente se ha publicado la estandarización chilena de la Escala Wechsler de Inteligencia para Adultos-cuarta edición (Coalson y Raiford, 2008; Rosas et al., 2013; Wechsler, 2008). Esta prueba se presenta en un momento de transformación de la cultura de evaluación del país y constituye la primera actualización que se realiza al instrumento desde la década de los sesenta.

El país también se está transformando socialmente y el escenario actual concentra la mayoría de la población en las zonas urbanas (Instituto Nacional de Estadística [INE], 2003a). Sin embargo, queda una porción del país que sigue residiendo en zonas rurales, dedicada al trabajo del campo y con acceso restringido a nuevas oportunidades o experiencias de alta calidad educativa (Organización para la Cooperación Económica y el Desarrollo [OCDE], 2010; Organización de Estados Iberoamericanos [OEI], 2001). En este escenario, la presente investigación ha explorado las diferencias en el rendimiento en WAIS-IV, estandarizado en Chile, para un grupo de jóvenes donde se consideró la zona de residencia y el nivel educativo de los padres como variables de interés. Se busca entregar elementos que enriquezcan el juicio de los/as psicólogos/as que realizan diagnósticos cognitivos con este instrumento, para favorecer una mejor interpretación de sus resultados.

\section{El concepto de ruralidad}

El concepto de ruralidad surgió a partir de una diferenciación con "lo urbano". Se ha propuesto que "lo urbano corresponde al contexto de la ciudad, a una sociedad industrial o a un proceso civilizador de cambio del ecosistema natural a uno artificial y con una directa intervención del ser humano" (Lugo, s.f; p. 2).Lo rural se tiende a entender como "lo que aún no es urbano" (Baigorri, 1995; p.2).

Sin embargo, lo rural no se limita a esta definición. Pérez (2001) propone la ruralidad como un conjunto de regiones y de zonas (un territorio) cuya población desarrolla diversas actividades o se desempeña en distintos sectores como la agricultura, la artesanía, las industrias pequeñas y medianas, el comercio, los servicios, la ganadería, la pesca, la minería, la extracción de los recursos naturales y el turismo, entre otros. En dichas zonas existen asentamientos que se relacionan entre sí y con el exterior, en los cuales interactúan una serie de instituciones públicas y privadas.

Para efectos prácticos, la ruralidad suele definirse a partir de la cantidad de habitantes y las actividades económicas predominantes. En el caso de Chile, el INE define como una entidad rural a aquellos asentamientos humanos concentrados o dispersos en el que habitan menos de 2.000 personas, con menos del 50\% de su población económicamente activa dedicada a actividades secundarias $y / o$ terciarias (INE, 2004). Para el país, según lo indicado por el censo del año 2002, el $13.4 \%$ de la población habita en zonas rurales, en tanto que en las zonas urbanas vive el $86.6 \%$ de la población total (INE, 2003a). La región del Biobío presenta un porcentaje de ruralidad de 17.9\% (INE, 2003b), siendo la séptima región con mayor porcentaje de ruralidad del país (INE, 2003a).

Respecto al rendimiento académico, varias investigaciones han demostrado diferencias de desempeño entre jóvenes rurales y urbanos. Por ejemplo, el Segundo Estudio Regional Comparativo y Explicativo [SERCE] y el Programa Internacional para la Evaluación de Estudiantes [PISA] (Murillo y Román, 2009; OCDE, 2010). Las pruebas realizadas en estos estudios exponen diferencias significativas entre el rendimiento de los residentes en zonas rurales versus los residentes de zonas urbanas e hipotetizan que tales diferencias son consecuencia de la inequidad educativa propia de los sectores rurales. Según los criterios de la 
OCDE (2010), la diferencia equivaldría a más de un año de educación formal.

Las conclusiones derivadas de estos resultados, explican que los estudiantes de escuelas ubicadas en una ciudad tienen acceso a recursos que potencian el aprendizaje, como bibliotecas y museos, a diferencia de aquellos que habitan en un sector rural. Más aún, en comunidades densamente pobladas, los colegios pueden contar con una mayor cantidad de recursos educacionales de mejor calidad, mientras que sectores más aislados deben contar con apoyo focalizado o políticas educacionales especiales para asegurarse que sus alumnos logren su potencial (OCDE, 2010).

En Chile, el 79.1\% de las escuelas ubicadas en sectores rurales son de dependencia municipal (Moreno, 2007). Estas escuelas obtienen sistemáticamente puntajes más bajos en la Prueba de Selección Universitaria (PSU) y en el Sistema de Medición de la Calidad de la Educación (SIMCE), en comparación con las de dependencia privada o subvencionada (Campos, 2010). Con estos datos es sencillo sugerir que hay una segregación en el país en cuanto al acceso educacional en sectores rurales. Más allá de esto, los municipios rurales tienden a recibir menos recursos del Estado por la baja matrícula que poseen y, en consecuencia, muchas veces tienen dificultades para satisfacer necesidades educativas en las escuelas que dependen de ellos (Moreno, 2007).

\section{La deprivación sociocultural como elemento central}

La inequidad descrita en el marco del contexto rural se enmarca bajo el concepto de deprivación sociocultural. Ésta es entendida como la carencia -por razones internas o por condiciones ambientales- de la transmisión cultural, que reduce la capacidad de las personas para modificar sus estructuras cognitivas y responder adecuadamente a las fuentes de estimulación (Barello, Palmucci y Schuager, 2007). Hace referencia a un estado de desventaja que es tanto observable como demostrable en relación con la comunidad, la sociedad o la nación a la cual pertenece un individuo, una familia o un grupo (Townsend, Phillimore y Beattie, 1988), como consecuencia de la falta de recursos (Social Disadvantage Research Centre, 2003).

En América Latina, los esfuerzos de planificación social y económica se han concentrado en el desarrollo de las zonas urbanas, en desmedro de las zonas rurales. Éstas han pasado a depender de las grandes metrópolis, con dificultades en aspectos relativos al acceso de bienes y servicios. Esto ha llevado a que la mayor parte de la población rural de Latinoamérica se encuentre en condiciones de deprivación sociocultural (Viñas-Román, 2003).
Se han descrito como principales dificultades de las zonas rurales de Latinoamérica las condiciones socioeconómicas propias de este medio, la pobreza de las familias, el trabajo prematuro de niños, difícil acceso a las escuelas o carencia de ellas, infraestructura física precaria, continuos desplazamientos de la familia o de los padres. Se destaca también la situación sanitaria y sociocultural, la desnutrición infantil, la elevada mortalidad, las malas condiciones de la vivienda y del saneamiento ambiental, el analfabetismo de padres y familiares, las limitaciones de la comunicación en el hogar y la diferencia entre los valores de la familia y su medio y los de la escuela (Viñas-Román, 2003).

La investigación ha demostrado que la deprivación sociocultural incide en el desarrollo de las capacidades intelectuales de los individuos (De Zubiria, 2002). En niños que nacen y crecen en ambientes deprivados, tienden a verse afectadas conductas como la curiosidad, lo que incide en una baja motivación por las actividades académicas, dificultades atencionales en clases y baja comprensión de las explicaciones del profesorado. Ante esto, los alumnos desarrollan bajas expectativas y un sentimiento de incapacidad frente al logro académico, además de carencia de claves para una autorregulación efectiva del trabajo (Navarro, 2002), lo que dificulta la adquisición de los contenidos y competencias del currículum escolar. De esta forma, los ambientes socioculturalmente deprivados pueden generar trabas para la adquisición de habilidades cognitivas y lingüísticas, que se manifiestan en un retraso en el desarrollo de habilidades verbales y el pensamiento simbólico (Guevara-Benítez y Macotela-Flores, 2002; Navarro, 2002).

Se ha visto que las habilidades verbales son reforzadas en el hogar. Las madres de niños residentes en zonas de vulnerabilidad social, tienden a utilizar un vocabulario más simple (Contreras y Soriano, 2007), cargado de modismos y abundante presencia de incorrecciones léxicas y semánticas, situación que conduce a un desarrollo verbal atípico en los niños. A estas características se suma que, en la población rural, se ha observado una notoria apatía por el lenguaje escrito, especialmente los adultos, quienes no suelen otorgarle relevancia a este medio para comunicarse (Santana y Martín, 1993).

Estas características confluyen en un ambiente lingüístico deficiente, que impacta negativamente el desarrollo del lenguaje del niño, y a su vez compromete el desarrollo de las funciones ejecutivas. Bajo este contexto, es posible suponer que vienen asociadas dificultades escolares como consecuencia del retraso en la adquisición de habilidades y precursores que deben estar presentes para lograr el curso esperado de aprendizajes (Contreras y Soriano, 2007). 
Al comparar los resultados de jóvenes de zonas urbanas y jóvenes de zonas rurales en la Batería Woodcock de Proficiencia en el Idioma (Woodcock, 1981), que evalúa el desarrollo del lenguaje comprensivo, se evidencian diferencias significativas a favor de los estudiantes de las zonas urbanas, mientras que los estudiantes del área rural obtienen menores resultados en subpruebas que miden el conocimiento del significado de las palabras, lenguaje oral, receptivo y expresivo, identificación de letras y palabras, análisis fonético y estructural para pronunciar palabras que no son familiares, comprensión y vocabulario (Matalinares et al., 2007).

Asimismo, los jóvenes de las zonas rurales muestran un menor nivel de logro, en comparación con los urbanos, en memoria auditiva inmediata, rendimiento expresado en las áreas numérica, lógica y asociativa, habilidades estrechamente relacionadas con el lenguaje comprensivo (Matalinares et al., 2007).

Ante las diferencias descritas entre sectores rurales y urbanos, la presente investigación pretende caracterizar el desempeño de la población rural en pruebas estandarizadas de inteligencia, en comparación con sus símiles urbanos. A partir de la evidencia encontrada, se hipotetizó que la muestra rural obtendría un desempeño general más bajo que la muestra urbana, y que ésta estaría marcada por diferencias estadísticamente significativas en el Índice de Comprensión Verbal ${ }^{1}$.

\section{Método}

\section{Participantes}

En este estudio participaron voluntariamente cuarenta y cuatro jóvenes, con edades comprendidas entre los 16 y 19 años $(M=17.94 ; \mathrm{DE}=1.14)$, de la región del Biobío, elegidos a partir de un muestreo intencionado. La muestra estuvo diferenciada en dos grupos: grupo rural y grupo urbano. Los sujetos fueron estratificados según sexo y nivel educacional de los padres. Este último correspondió al promedio de años alcanzado en el sistema educativo formal por ambos padres de los jóvenes. En caso de que hubiera sólo un padre que reportara datos, se consideró la educación de éste como indicador. Según la cantidad de años de educación formal,

1 El índice de comprensión verbal está compuesto por tres subpruebas centrales (analogías, vocabulario e información) y una subprueba suplementaria (comprensión). la muestra se estratificó en tres niveles: básico ( 8 años), medio ( 9 a 12 años) y superior (13 años en adelante).

Grupo rural. Estuvo compuesto por veinte jóvenes (13 hombres y 7 mujeres), con edades comprendidas entre los 16 y 19 años $(M=17.73 ; \mathrm{DE}=1.14)$, que cumplieron las siguientes características: (1) haber habitado en sectores rurales de la región del Biobío por un periodo mínimo de 2 años previos al momento en que se realizó la evaluación y (2) estar cursando, en la fecha del estudio, segundo, tercero o cuarto año medio. Por conveniencia, esta muestra se extrajo de dos liceos: 10 de los jóvenes seleccionados pertenecían a un liceo científico humanista, ubicado en la localidad de Río Claro, y 14 de ellos a un liceo técnico agrícola, ubicado en la ciudad de Chillán. Este último liceo recibe estudiantes de localidades rurales de toda la región (Tabla 1).

Grupo urbano. Estuvo compuesto por veinticuatro jóvenes (13 hombres y 11 mujeres), con edades comprendidas entre 16 y 19 años $(\mathrm{M}=18.12 ; \mathrm{DE}=1.14)$, pertenecientes a sectores urbanos de la provincia de Concepción. Los criterios de inclusión fueron: (1) vivir en un sector urbano de la VIII región por al menos dos años y (2) estudiar en un establecimiento educacional de un sector urbano. Los datos de esta muestra fueron proporcionados por la comisión de estandarización de WAIS-IV, en la región del Biobío (Tabla 1).

Tabla 1. Datos sociodemográficos.

\begin{tabular}{lcccc}
\hline & Básico & Medio & Superior & Total \\
\hline Urbano & 8 & 4 & 12 & 24 \\
Rural & 4 & 14 & 2 & 20 \\
Total & 14 & 19 & 15 & 44 \\
\hline
\end{tabular}

\section{Instrumento}

Se utilizó la Escala Wechsler de Inteligencia para Adultos - cuarta edición, versión chilena (WAIS-IV, Rosas et al., 2013). Al momento del estudio, esta versión se encontraba en proceso de estandarización a cargo de la Pontificia Universidad Católica de Chile, en el marco del proyecto FONDEF D09I1238, “Construcción y Estandarización de Instrumentos de Evaluación Psicométrica para el Desarrollo de una Cultura de Evaluación Ética y Rigurosa" (Rosas y Cumsille, 2010).

WAIS-IV tiene por objeto evaluar las habilidades cognitivas y contempla cuatro factores: comprensión verbal, razonamiento perceptual, memoria de trabajo y velocidad de procesamiento (Lichtenberger y Kaufman, 2009). La prueba entrega resultados en términos de coeficiente intelectual total e índices respecto a cada factor mediante puntajes equivalentes usados para comparar al sujeto con respecto a 
su población. WAIS-IV consta de 10 subpruebas centrales y cinco suplementarias, que en conjunto arrojan el perfil cognitivo del sujeto que da cuenta del nivel de desarrollo de las dimensiones cognitivas consideradas.

Adicional a esto se aplicó a todos los sujetos un cuestionario de datos sociodemográficos para obtener su información básica, además de los años de escolaridad de sus padres, variable de interés para el estudio.

\section{Procedimiento}

La muestra rural fue contactada con previo consentimiento de los directivos de cada establecimiento educacional, quienes además proporcionaron los datos sociodemográficos de los estudiantes. Posterior a esto se entregó un consentimiento informado a cada estudiante con datos sobre la investigación e invitándolo a participar. En el caso de menores de 18 años, padres o apoderados también firmaron un consentimiento informado.

El instrumento se administró en un espacio libre de estímulos distractores dentro de sus respectivos establecimientos educacionales, en horario de clases, entre octubre y noviembre de 2011. La duración de cada sesión de evaluación fluctuó entre una hora con treinta minutos y dos horas. Los datos de la muestra urbana fueron proporcionados por la comisión de estandarización de WAIS-IV, en la región del Biobío.

\section{Análisis de datos}

Se consideró la inclusión de datos descriptivos y se realizó un Análisis de Varianza (ANOVA) para comparar las diferencias entre ambos grupos. Se utilizó el promedio de años de educación formal de los padres como variable proxy del nivel socioeconómico por la dificultad metodológica para caracterizar esta variable. Esta estrategia metodológica es avalada teóricamente por otras investigaciones (Mizala, Romaguera y Reinaga, 1999) y ha sido utilizada en otros estudios empíricos (e.g. Adimark, 2010). Se generaron tres categorías en base al nivel educacional de los padres: básico (0-8 años), medio (9-12 años) y superior (>13 años). Estas categorías fueron incluidas en los análisis realizados. Todos los análisis se condujeron con SPSS v.19 para interpretación de resultados y se fijó el punto de significancia estadística en .05 (Fisher, 1956).

\section{Resultados}

Los resultados ponen en evidencia un rendimiento promedio superior del grupo urbano en el Coeficiente Intelectual Total (CIT) y los cuatro índices factoriales. Las diferencias en todos los casos ubican el rendimiento obtenido por el grupo urbano por sobre el rendimiento alcanzado por el grupo rural (Figura 1).

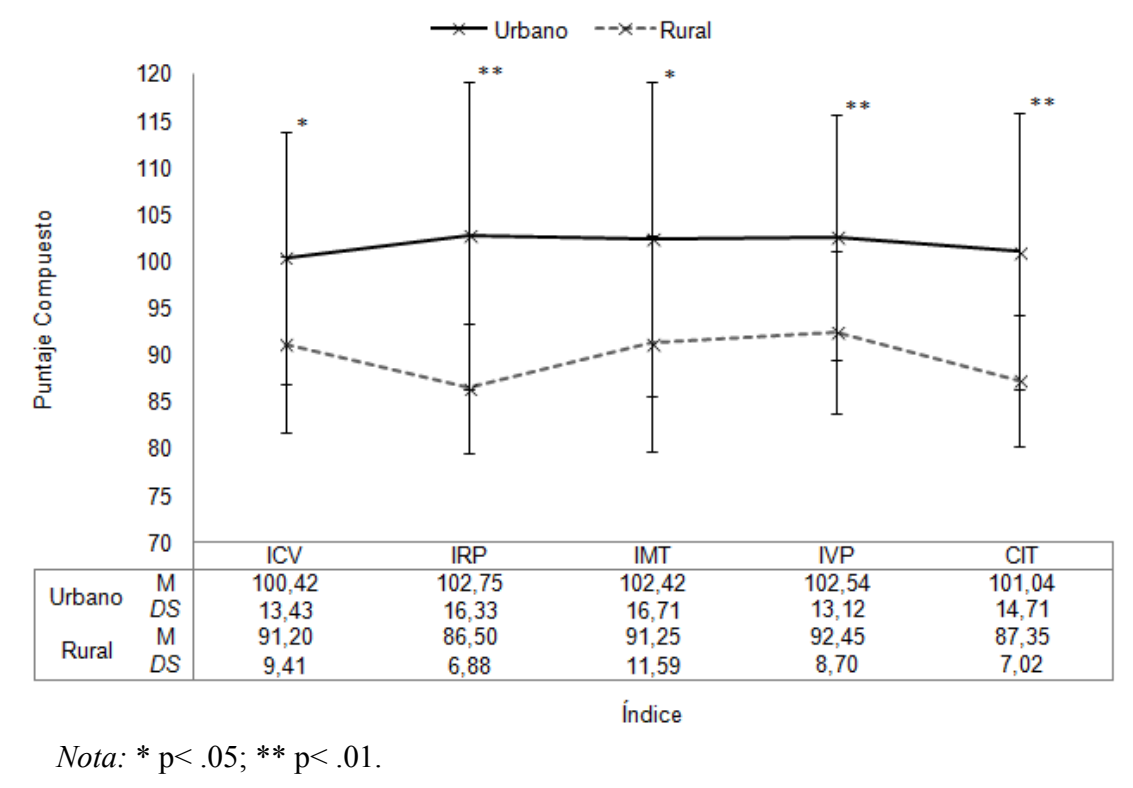

Figura 1. Medias de indices factoriales y coeficiente intelectual total. 
Para efectos comprensivos, el análisis de resultados se organiza de lo general a lo particular. En el CIT se observa una diferencia promedio estadísticamente significativa de 14 puntos entre los dos grupos, a favor del grupo urbano $\left(\mathrm{F}[2,43]=14.53 ; p<.01 ; \eta^{2}=0.26\right)$. Al incorporar en el modelo el nivel educativo de los padres como variable relevante, se registra una interacción significativa $(\mathrm{F}[2,43]=6.00 ; p<$ $\left..01 ; \eta^{2}=0.24\right)$. En esta interacción se observa que el CIT varía en función del nivel educativo de los padres y la zona de residencia. Así, para el nivel educativo básico, no hay diferencias entre los grupos $\left(\mathrm{F}[2,11]=0.05 ; p=.83 ; \eta^{2}=\right.$ $.01)$, mientras que el grupo urbano supera al rural en los niveles medio $\left(\mathrm{F}[2,17]=17.83 ; p<.01 ; \eta^{2}=0.53\right)$ y superior $\left(\mathrm{F}[2,13]=13.73 ; p<.01 ; \eta^{2}=0.53\right)$.

$\mathrm{Al}$ analizar tendencias intragrupales, se observa que para el grupo urbano hay diferencias significativas entre los niveles básico-medio $(p<.01)$ y básico-superior $(p<.01)$. No se observa diferencia entre los niveles medio-superior. Para el grupo rural en cambio, existe homogeneidad entre los niveles educativos (Figura 2).

Los índices factoriales representan el siguiente nivel de análisis. En el Índice de Comprensión Verbal (ICV) se registra una diferencia de nueve puntos en promedio entre los grupos. Esta diferencia es estadísticamente considerable $\left(\mathrm{F}[2,43]=6.68 ; p=.01 ; \eta^{2}=0.14\right)$. Al incorporar el nivel educativo de los padres al modelo, se observa una diferencia estadísticamente significativa entre los grupos únicamente para el nivel medio $\left(\mathrm{F}[2,17]=6.14 ; p<.01 ; \eta^{2}=0.28\right)$.

A nivel intragrupo se observan diferencias en el grupo urbano entre el nivel de educación básico y medio $(p=.04)$ y entre los niveles básico y superior $(p<.01)$. No existe diferencia entre los niveles medio y superior. En el grupo rural existe una diferencia estadísticamente significativa entre los niveles medio y superior $(p=.04)$, no así entre básico y superior. La diada básico-medio tampoco presenta diferencias notorias (Figura 3).

Para el Índice de Razonamiento Perceptual (IRP), los grupos presentan una diferencia promedio estadísticamente significativa de 16 puntos $\left(\mathrm{F}[2,43]=17.21 ; p<.01 ; \eta^{2}=0.29\right)$. $\mathrm{Al}$ incorporar el nivel educativo de los padres se observa interacción entre ambas variables $(\mathrm{F}[2,43]=3.88 ; p=.03$; $\eta^{2}=0.17$ ), lo que daría cuenta de un comportamiento distinto de los puntajes del factor, en función de la zona de residencia y el nivel educativo de los padres. Se aprecian diferencias estadísticamente considerables entre los grupos para los niveles medio $\left(\mathrm{F}[2,17]=31.99 ; p<.01 ; \eta^{2}=0.67\right)$ y superior $\left(\mathrm{F}[2,13]=6.34 ; p=.03 ; \eta^{2}=0.35\right)$, no así para el nivel básico.

Se aprecian diferencias entre los niveles básico y medio $(\mathrm{p}=.01)$ y los niveles básico y superior $(p<0.01)$ para el grupo

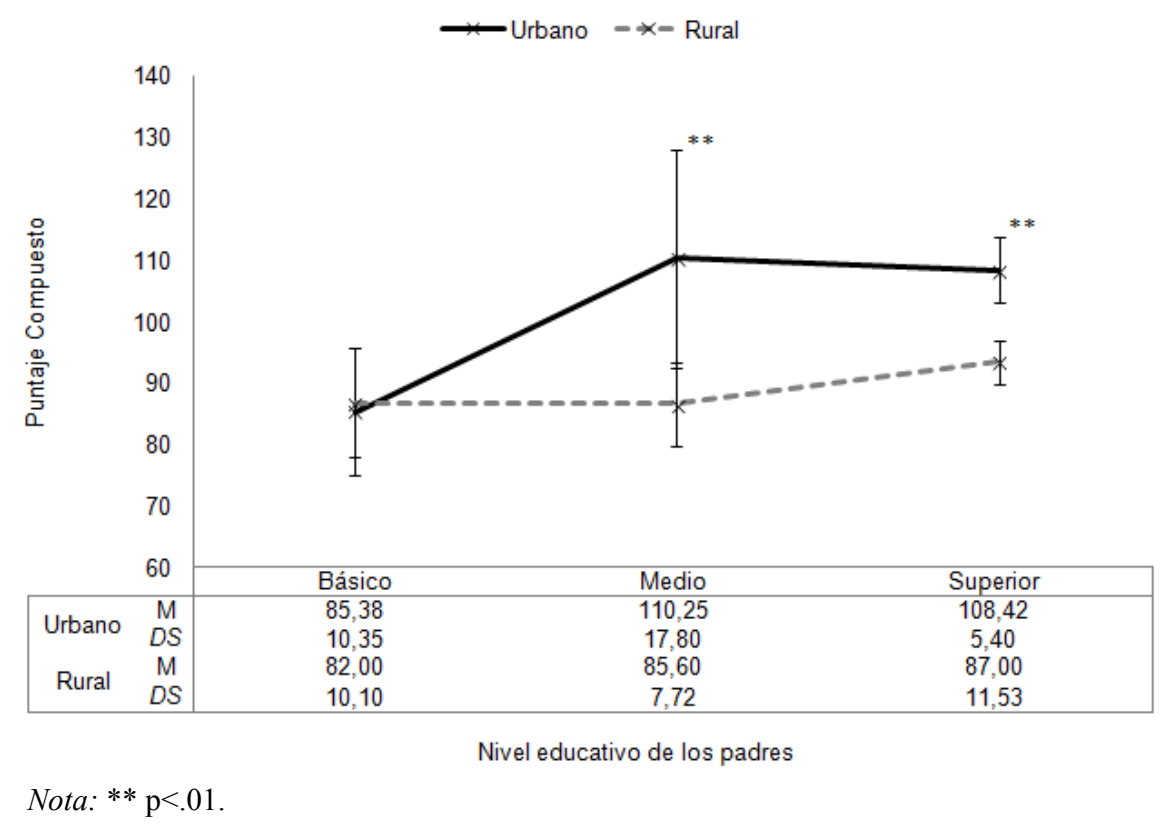

Figura 2. Medias CIT segmentadas por nivel educativo de los padres. 


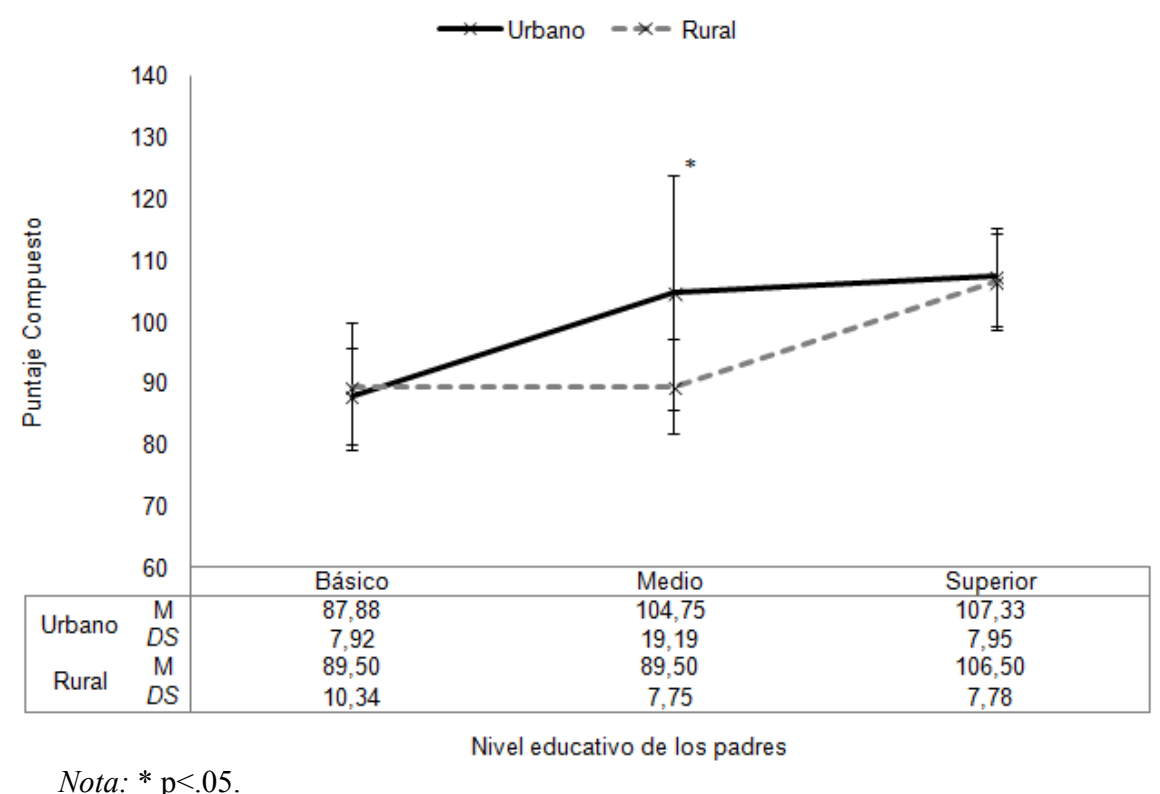

Figura 3. Medias ICV segmentadas por nivel educativo de los padres.

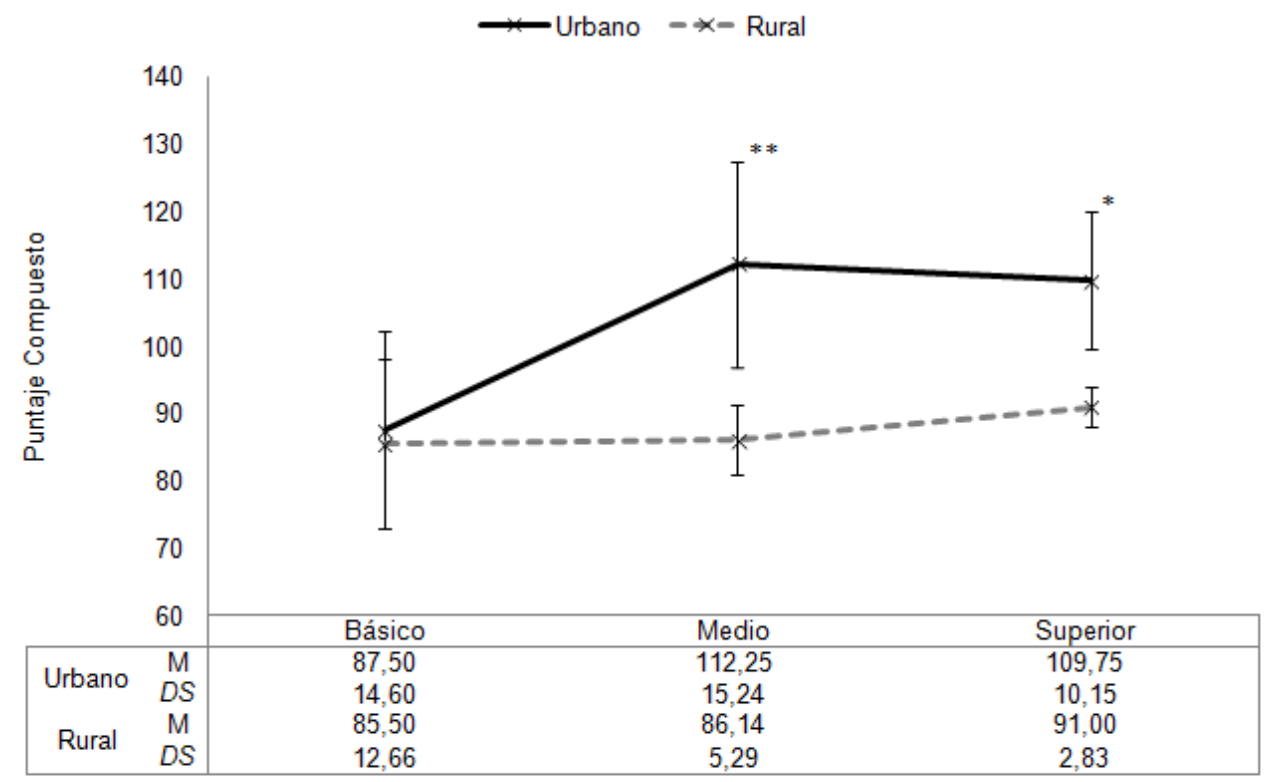

Nivel educativo de los padres

Nota: $* p<.05 ; * * p<.01$.

Figura 4. Medias IRP segmentadas por nivel educativo de los padres.

urbano. El grupo rural se comporta de forma homogénea (Figura 4).

El Índice de Memoria de Trabajo (IMT) registra una diferencia significativa de 11 puntos entre los grupos a favor del grupo urbano $\left(\mathrm{F}[2,43]=6.37 ; p=.02 ; \eta^{2}=0.13\right)$. Al comparar los grupos por nivel educacional de los padres, se observa una diferencia estadísticamente significativa sólo para el nivel medio $\left(\mathrm{F}[2,17]=8.54 ; p=.01 ; \eta^{2}=0.35\right)$.
A nivel intragrupal se aprecia homogeneidad en los resultados de ambos grupos, entre los distintos niveles educacionales de los padres (Figura 5).

El Índice de Velocidad de Procesamiento (IVP) presenta una diferencia de medias de 10 puntos a favor del grupo urbano $\left(\mathrm{F}[2,43]=8.64 ; p<.01 ; \eta^{2}=0.17\right)$. Asimismo, se observa una interacción entre las variables al incluir el nivel educativo de los padres en el análisis $\left(\mathrm{F}[2,43]=6.85 ; p<.01 ; \eta^{2}=0.27\right)$, 


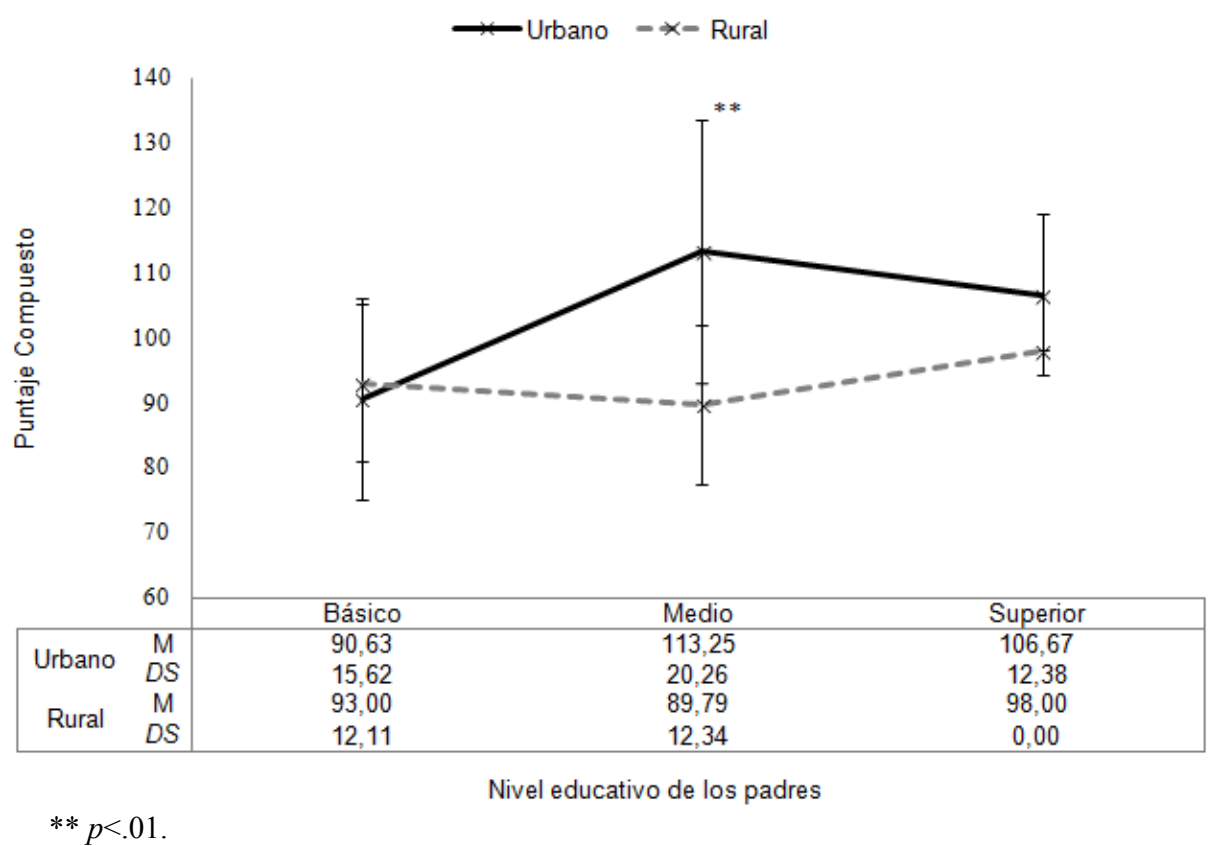

Figura 5. Medias IMT segmentadas por nivel educacional de los padres.

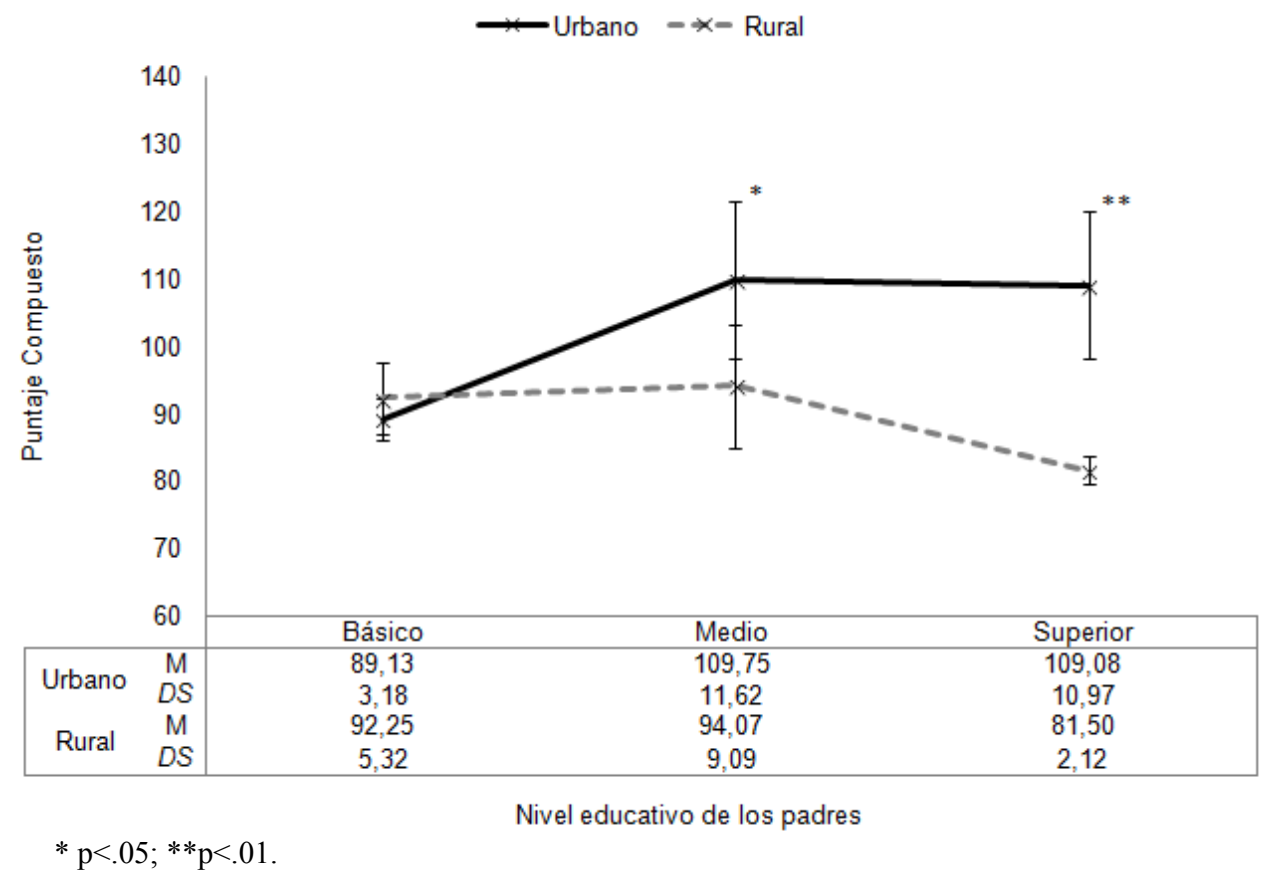

Figura 6. Medias IVP segmentadas por nivel educativo de los padres.

lo que implica que el IVP varía en función de la zona de residencia y el nivel educativo. De esta forma, se aprecian diferencias estadísticamente significativas entre las medias de ambos grupos para los niveles medio $(\mathrm{F}[2,17]=8.28 ; p=.01$; $\left.\eta^{2}=0.34\right)$ y superior $\left(\mathrm{F}[2,13]=11.79 ; p<.01 ; \eta^{2}=0.50\right)$, y no para el nivel básico de educación de los padres.
Intragrupo: se observan diferencias en el grupo urbano entre los niveles básico y medio $(p<.01)$ y entre los niveles básico y superior $(p<.01)$ de educación de los padres. El grupo rural se comporta de forma homogénea (Figura 6).

A nivel de subprueba, se observa una diferencia estadísticamente significativa a favor del grupo urbano en 10 
Tabla 2. Descriptivos por subprueba

\begin{tabular}{llccccc}
\hline & & \multicolumn{2}{c}{ Grupo Urbano } & \multicolumn{2}{c}{ Grupo Rural } \\
& Subprueba & $M$ & $D S$ & $M$ & $D S$ & $p$ \\
\hline ICV & Analogías & 9.79 & 2.60 & 8.60 & 2.28 & \\
& Vocabulario & 9.58 & 2.84 & 8.45 & 1.96 & $* *$ \\
& Información & 10.17 & 3.10 & 7.45 & 1.47 & $*$ \\
& Comprensión & 10.08 & 2.57 & 8.25 & 2.10 & $* *$ \\
IRP & Construcción con cubos & 11.04 & 3.32 & 7.80 & 1.82 & $* *$ \\
& Matrices de razonamiento & 10.00 & 3.12 & 7.25 & 2.07 & $* *$ \\
& Rompecabezas visuales & 10.17 & 3.23 & 7.80 & 1.67 & $*$ \\
& Balanzas & 10.42 & 3.62 & 8.30 & 2.06 & \\
& Figuras incompletas & 9.58 & 2.73 & 9.30 & 2.41 & \\
& Retención de dígitos & 10.12 & 3.56 & 8.70 & 2.27 & $*$ \\
& Aritmética & 10.38 & 3.61 & 7.90 & 2.20 & $*$ \\
& Secuenciación letras-números & 10.38 & 2.87 & 9.20 & 2.07 & $*$ \\
& Búsqueda de símbolos & 10.29 & 2.07 & 8.45 & 2.04 & $*$ \\
& Claves & 10.00 & 3.01 & 8.15 & 1.84 & $*$ \\
& Cancelación & 8.42 & 2.92 & 6.20 & 2.17 & $* *$ \\
\hline
\end{tabular}

Nota: $* \mathrm{p}<.05 ; * * \mathrm{p}<.01$.

de las 15 subpruebas. Entre éstas destacan por un mayor tamaño de efecto: construcción con cubos $(\mathrm{F}[2,43]=15.23$; $\left.p<.01 ; \eta^{2}=0.27\right)$, información $\left(\mathrm{F}[2,43]=12.89 ; p<.01 ; \eta^{2}=0.24\right)$, matrices de razonamiento $\left(\mathrm{F}[2,43]=11.33 ; p<.01 ; \eta^{2}=0.21\right)$, rompecabezas visuales $\left(\mathrm{F}[2,43]=8.77 ; p<.01 ; \eta^{2}=0.17\right)$, búsqueda de símbolos $\left(\mathrm{F}[2,43]=8.74 ; p<.01 ; \eta^{2}=0.17\right)$ y cancelación $\left(\mathrm{F}[2,43]=8.00 ; p<.01 ; \eta^{2}=0.16\right)($ Tabla 2$)$.

\section{Discusión}

Las diferencias en el rendimiento en pruebas estandarizadas de medición de habilidades cognitivas entre zonas urbanas y rurales se explican principalmente por el impacto que genera habitar en ambientes socioculturalmente deprivados (De Zubiria, 2002; Guevara-Benítez y Macotela-Flores, 2002; Matalinares et al., 2007; Navarro, 2002). De igual forma se ha demostrado que un bajo nivel socioeconómico de las familias también afecta este rendimiento (Flores-Mendoza y Nascimiento, 2007; Roazzi y Campello, 2002).

En este estudio se demuestra que la relación entre zona de residencia y nivel socioeconómico con el desarrollo de habilidades cognitivas tiene una tendencia menos lineal que la hasta ahora hipotetizada. La interacción identificada a nivel de CIT evidencia que las habilidades cognitivas se ven influenciadas tanto por la zona de residencia como por el nivel socioeconómico de las familias. De esta manera, el efecto del nivel socioeconómico sobre ciertas habilidades cognitivas va a depender del lugar donde habite el sujeto. En el nivel socioeconómico bajo no se observan diferencias entre los grupos urbano y rural, posiblemente por estar ambos sectores en condiciones de deprivación. Éste no es el caso de los sectores más acomodados, donde habitar en una zona rural tiene un impacto negativo sobre las habilidades cognitivas de los jóvenes, observándose diferencias significativas en su rendimiento intelectual en comparación al de jóvenes de la misma condición socioeconómica que habitan en sectores urbanos.

Asimismo, los resultados obtenidos sugieren que algunas habilidades cognitivas tienen una correlación más fuerte con el nivel socioeconómico de pertenencia de las familias. Estas habilidades están específicamente ligadas al área verbal y la memoria de trabajo. Investigaciones previas han demostrado un mayor efecto del nivel socioeconómico en el constructo de inteligencia cristalizada con respecto a la inteligencia fluida (Cattell, 1987), constructo que tiene estrecha relación con el ICV de WAIS-IV (Lichtenberger y Kaufman, 2009), por lo que el mayor efecto del nivel socioeconómico de los padres debiera estar reflejado en este factor.

Por su parte, la memoria de trabajo, tal y como se explora en este batería, tiene una fuerte dependencia del componente verbal pues la presentación de estímulos requiere de la activación del loop fonológico (Carroll, 1993; Hernández, Díaz, Jiménez, Martín, Rodríguez y García, 2012; Lichtenberger y Kaufman, 2009).

Como limitación de este estudio, cabe destacar el bajo tamaño de la muestra y la falta de homogeneidad en la distribución de ésta en los distintos niveles socioeconómicos. Es deseable que investigaciones como ésta puedan replicarse con muestras de mayor tamaño para favorecer datos más generalizables sobre las diferencias en habilidades cognitivas entre sectores urbanos y rurales de Chile. Además, dada la alta diversidad de entornos rurales en el país (San Miguel, 
2005), se espera que dichas muestras puedan desprenderse desde distintas localidades y contextos rurales.

Resulta interesante la posibilidad de que esta investigación favorezca aproximaciones futuras hacia los sectores rurales y genere acercamientos que propicien mejorías contextualizadas a cada cultura rural, tanto en la calidad educativa como a nivel socioeconómico, para progresivamente disminuir el impacto que produce la brecha entre la población rural y urbana.

\section{Referencias}

Adimark. (2010). Mapa socioeconómico de Chile. Santiago, Chile: Adimark.

Baigorri, A. (1995). De lo rural a lo urbano. En Congreso Español de Sociología, Horizontes desde la incertidumbre. Simposio efectuado en el V Congreso Español de Sociología, Granada, España.

Barello, A., Palmucci, V., y Schuager, N. (2007). Reflexión Teórica: “De novatos a expertos del pensar: La teoría de la modificabilidad estructural cognitiva”. Recuperado de http://www.xpsicopedagogia.com. ar/de-novatos-a-expertos-del-pensar-la-teoria-de-la-modificabilidadestructural-cognitiva.html

Campos, J. (2010). Las desigualdades educativas en Chile. Buenos Aires: Foro Latinoamericano de Políticas Educativas.

Carroll, J. B. (1993). Human cognitive abilities. A survey of factor-analytic studies. New York: Cambridge University Press.

Cattell, R. (1987). Intelligence: Its structure, growth, and action. Amsterdam: North-Holland.

Coalson, D. L., y Raiford, S.E. (2008). Wechsler Adult Intelligence ScaleFourth Edition technical and interpretative manual. San Antonio, TX: Psychological Corporation.

Contreras, M., y Soriano, M. (2007). Morfología flexiva en el trastorno específico del lenguaje y en la deprivación cultural. Revista de Logopedia, Foniatría y Audiología, 3, 110-117.

De Zubiria, J. (2002). Teorías contemporáneas de la inteligencia y la excepcionalidad. Bogotá: Magisterio.

Fisher, R. A. (1956). Statistical methods and scientific inference. New York: Hafner.

Flores-Mendoza, C. y Nascimiento, E. (2007). Condição cognitiva de crianças de zona rural. Estudios de Psicología, 1, 13-22.

Guevara-Benítez, Y., y Macotela-Flores, S. (2002). Sondeo de habilidades preacadémicas en niños y niñas mexicanos de estrato socioeconómico bajo. Revista Interamericana de Psicología, 36, 177-255.

Hernández, S., Díaz, A., Jiménez, J., Martín, R., Rodríguez, C., y García, E. (2012). Datos normativos para el test de Span Visual: Estudio evolutivo de la memoria de trabajo visual y la memoria de trabajo verbal. European Journal of Education and Psychology, 5(1), 65-77.

Instituto Nacional de Estadísticas - Chile (2003a). Censo 2002: síntesis de resultados. Santiago: INE.

Instituto Nacional de Estadísticas - Chile (2003b). Censo 2002: Síntesis de resultados. - Región del Bío Bío. Santiago: INE.

Instituto Nacional de Estadísticas - Chile (2004). Chile: Estimaciones $y$ proyecciones de población por sexo y edad. País urbano-rural. Santiago, Chile: Autor.

Lichtenberger, E., y Kaufman, A. (2009). Essentials of WAIS-IV assessment. Nueva Jersey: John Wiley y Sons.
Lugo, P. (s.f). El paisaje urbano. Recuperado de http://www.academia. edu/1169181/EL_PAISAJE_URBANO

Matalinares, M., Dioses, A., Ārenas, C., Díaz, G., Chávez, J., Yaringaño, J., y Suárez, J. (2007). Lenguaje comprensivo y memoria auditiva inmediata en estudiantes de $5 .{ }^{\circ}$ y $6 .{ }^{\circ}$ grado de primaria de la zona rural y urbana de Lima. Revista IIPSI, 2, 71-83.

Mizala, A., Romaguera, P., y Reinaga, T. (1999). Factores que inciden en el rendimiento escolar en Bolivia. Santiago: Universidad de Chile, Centro de Economía Aplicada.

Moreno, C. (2007). Las escuelas rurales en Chile: La municipalización y sus fortalezas y debilidades. Revista Digital y Rural, 8, 1-6.

Murillo, F., y Román, M (2009). Mejorar el desempeño de los estudiantes de América Latina. Algunas reflexiones a partir de los resultados del SERCE. Revista Mexicana de Investigación Educativa, 14(41), 451-484.

Navarro, J. (2002, enero). La compensación educativa: Marco, realidad y perspectivas. En: Formación específica en compensación educativa e intercultural para agentes educativos. Curso efectuado en Consejería de Educación y Cultura, Murcia, España.

Organización de Estados Iberoamericanos. (2001). Organización y perspectivas del nivel inicial en Iberoamérica: Chile. Madrid: Autor.

Organización para la Cooperación Económica y el Desarrollo (2010). PISA 2009 Results: Overcoming social background - equity in learning opportunities and outcomes (Volume II). Doi:10.1787/9789264091504-en

Pérez, E. (2001). Hacia una nueva visión de lo rural. En: N. Giarracca (Comp.). ¿Una nueva ruralidad en América Latina? (pp. 17-29). Buenos Aires: CLACSO.

Roazzi, A., y Campello, B. (2002). Repensando a inteligencia. Paidéia, 12, 31-55.

Rosas, R., Tenorio, M., Pizarro, M., Cumsille, P., Bosch, A., Arancibia, S., Carmona, M., Pérez-Salas, C. P., Pino, E., Vizcarra, B., y ZapataSepúlveda, P. (2013). Estandarización de la Escala Wechsler de Inteligencia para Adultos-Cuarta Edición en Chile: descripción de la prueba, procesos de adaptación, procedimiento de generación de normas y evidencia psicométrica. Sometido Psykhe.

Rosas, R., y Cumsille, P. (2010). Construcción y estandarización de instrumentos de evaluación psicométrica para el desarrollo de una cultura de evaluación ética y rigurosa (Proyecto FONDEF D09I1238). Santiago de Chile: Pontificia Universidad Católica, Escuela de Psicología.

San Miguel, J. (2005). Nuevas demandas de la educación básica rural. Revista Digital y Rural, 5, 1-11.

Santana, M., y Martín, P. (1993). Módulo didáctico 2: La educación infantil en el medio rural. Andalucía: Junta de Andalucía, consejería de educación y ciencia.

Social Disadvantage Research Centre (2003). Scottish indices of deprivation 2003. Oxford: SDRC.

Strauss, E., Sherman, E., y Spreen, O. (1999). A compendium of neuropsichological test: administrations, norms, and commentary, third edition. Oxford: Oxford University Press.

Townsend, P., Phillimore, P., y Beattie, A. (1988). Health and deprivation: Inequality and the north. Nueva York: Crom Helm.

Viñas-Román, J. (2003). Transformar la educación rural en América Latina y el Caribe. Un desafío insoslayable. Revista Digital y Rural, $1,1-14$.

Wechsler, D. (1979). La medida de la inteligencia del adulto. Buenos Aires: Huascar.

Wechsler, D. (2008). Wechsler adult intelligence scale-fourth edition. San Antonio, TX: Pearson.

Woodcock, R. (1981). Woodcock Language Proficiency Battery, Spanish form. USA: Teaching Resources. 